\title{
Visuotactile Peripersonal Space in Healthy Humans: Evidence from Crossmodal Congruency and Redundant Target Effects
}

\author{
Wataru TERAMOTO ${ }^{1,2, *}$ and Tomoaki KAKUYA ${ }^{2}$ \\ ${ }^{1}$ Department of Psychology, Faculty of Letters, Kumamoto University, Kumamoto 860-8555, Japan \\ ${ }^{2}$ Department of Computer Science and Systems Engineering, Muroran Institute of Technology, \\ Muroran 050-8585, Japan
}

Received January 16, 2015; final version accepted May 11, 2015

\begin{abstract}
Single neuron studies on monkeys provided convincing evidence for the existence of visuotactile peripersonal space. The range of this space was operationally defined as a space where visuotactile interactions occurred at the neuronal level, and the distance between the body part and visual stimuli was a crucial factor. While the functional similarities in humans were mainly evidenced by studies with patients with right brain damage exhibiting extinction, less is known about the same in healthy adults. The present study demonstrated the existence of visuotactile peripersonal space in healthy adults using two psychophysical measurements. In Experiment 1, participants discriminated the location of vibrotactile target stimuli presented on their left or right hand, while trying to ignore visual distractors that were independently presented close to or away from the tactile stimuli, either on the same side as the target stimulus or on the opposite side (visuotactile congruency task). Results showed that crossmodal congruency effects were greater when visual stimuli were in proximity to the hands, rather than away from them. In Experiment 2, redundant target effects were measured by using a go/no-go paradigm where participants produced speeded responses all to randomized sequence of unimodal (visual or tactile) and simultaneous visuotactile targets presented in one hemispace, while ignoring tactile stimuli presented in the other hemispace. Visual targets were presented either close to or away from the hand. Results showed that the statistical facilitation model was violated (i.e., the coactivation model was supported) only when visual stimuli were presented in proximity to the stimulated hand. These results suggest that visuotactile peripersonal space was distinctly and modularly represented in healthy human brains.
\end{abstract}

KEYWORDS: visuotactile interaction, peripersonal space, crossmodal congruency effect, redundant target effect

\section{Introduction}

The space immediately surrounding a body part is of importance in interacting with objects in the environment. Many neurophysiological and neuropsychological studies have provided evidence that the space around a body part is represented as distinct space from personal and extrapersonal spaces (i.e., space very close to the body's surface and the space away from the body, respectively) in the brain [3, 6, 9, 18-20,29-31,33,42-45]. This space is called as the peripersonal space $[39,45]$. Studies on single-cell recordings in monkeys primarily provided evidence for the existence of bimodal neurons that responded not only to tactile stimuli on the surface of the body, but also visual stimuli presented close to the body surface $[3,9,18-20,42-45]$. The firing rate of the neurons was reported to be inversely proportional to the distance of visual stimuli from the body surface. The range of the peripersonal space was thus operationally defined as a space where visuotactile interactions occurred at the neuronal level [32]. Evidence for the existence of peripersonal space in humans was mainly provided by patients with right brain damage (RBD) exhibiting extinction $[6,29,30,33]$. Extinction is a phenomenon where patients cannot detect a contralesional target when it is presented on the ipsilesional as well as the contralesional sides; however, they can do the same when the target is presented on any one side [1]. This phenomenon can occur not only when the two targets are defined in a single sensory modality, but also when they are defined in two different modalities. For example, when a tactile target is presented on the contralesional side and a visual target is presented in proximity to the comparative body part on the ipsilesional side, the patients fail to detect the tactile target. More importantly, the distance between the visual target and the body surface is reported to be crucial $[6,29,30]([28]$ for a review). This crossmodal extinction is not pronounced when the visual target is presented away from the body surface. This phenomenon is considered as evidence for visuotactile peripersonal space in humans. 
However, only one study demonstrated the dependency of visuotactile interactions on the distance between the visual stimuli and the body surface (i.e., visuotactile peripersonal space) in healthy humans*. Sambo and Forster had their participants direct attention to either the participants' left or right hand and asked them to discriminate a specific pattern of tactile stimuli presented on the attended hand from tactile non-target stimuli, while ignoring the visual stimuli presented either in proximity to or far from the attended hand and all stimuli presented on the unattended side [46]. They also measured the event-related potentials in response to the tactile non-targets presented along with the task irrelevant visual stimuli. Their results showed that reaction times (RTs) were shorter when the task irrelevant visual stimuli were presented in proximity to the hand and on the same side of the tactile target, than when the visual stimuli were presented away from the hand. Furthermore, the P100 components recorded over and close to the somatosensory cortex were enhanced in the former condition as compared with those in the latter conditions. This study suggested that visuotactile peripersonal space is represented distinctively from extrapersonal space in the healthy human brain as well. The aim of the present study was to obtain further behavioral evidence for the discrete representations of visuotactile peripersonal space in healthy human adults. Specifically, the present study adopted the crossmodal congruency effects that appeared in the visuotactile congruency task and the redundant target effects (RTEs) normally observed in simple/ choice detection and go/no-go tasks.

In studies with a typical visuotactile congruency task, participants were asked to hold a foam cube in each of their left and right hands (e.g., [52]; [54] for a review). Two pair of tactile and visual stimulators are attached to the cube by the thumb and index finger. Participants were required to generate speeded elevation discrimination responses to a target presented in one sensory modality, while trying to ignore task irrelevant distractors presented in the other modality. The spatial relationship between visual and tactile stimuli is manipulated. Responses are slower when the target and distractor are presented on different sides (incongruent condition) than when both are presented on the same side, or when no distractor is presented. Some studies used the "congruency effect" as an index by subtracting RT for the congruent condition from those for the incongruent condition [53,54]. The visual distractors normally have a greater influence when they are presented on the same hand rather than on the other hand. As for the visuotactile peripersonal space, most previous studies manipulated the spatial discrepancy by changing the azimuthal locations between the visual and tactile stimuli [52], but not by manipulating depth or distance from the body part. Thus, the present study investigated the effect of the spatial discrepancy in the near-far dimension between visual and tactile stimuli, on the visuotactile interactions. This manipulation would be more comparable to single-cell recordings in monkeys.

Todd observed a reduction of RTs in detecting events when multiple sensory modalities were simultaneously stimulated, as compared with when only a single modality was stimulated [57]. Participants were asked to produce speeded responses when any target was presented, irrespective of the modality. In some trials, a target was presented on a single sensory modality, while in others targets were presented on more than one modality. RTs are shorter for the latter than the former conditions. This effect has been called as RTEs. The RTEs can be explained by two models: the race model (in other words, the separate activation or statistical facilitation model) and the coactivation model. In the race model, signals on multiple modalities are considered to produce separate activations in different channels. Responses are assumed to be produced by the faster of the two processes. Thus, RTs will be generally less when multiple modalities are simultaneously stimulated than when a single modality is stimulated [37]. This suggests that the system never combines activation from different channels. In contrast, the coactivation model assumes that the unimodal activations are first combined to jointly trigger a response in each single trial. It is assumed that the violation of the race model supports the coactivation model [37]. While several studies have shown the violation of the race model in visuotactile interactions $[8,10,16,17,22]$, no study has demonstrated the effects of spatial discrepancy in the depth between visual and tactile stimuli on visuotactile interactions using this paradigm. Forster et al. manipulated the distance of visual stimuli from the tactile stimuli on the hands to compare visuotactile interactions in the near-far dimension. Their participants extended their forearms that were rested above a table and tactile stimuli were presented on the tips of the little finger on the right or the left hand. There were three distance conditions for the visual stimuli: immediately around the hands $(57 \mathrm{~cm}$ from the body), between the hands and the body $(28.5 \mathrm{~cm}$ from the body and the hands), and $28.5 \mathrm{~cm}$ away from the hands (approximately $85.5 \mathrm{~cm}$ from the body). The participants conducted speeded detection of any stimuli irrespective of their position. Forster et al. found evidence for the violation of the race model in all the distance conditions [10], such that they failed to observe the dependency of visuotactile interactions on the

\footnotetext{
*Maravita, Spence, Sergant, and Driver [36] may be a relevant study in the current literature, although they did not directly show the dependency of visuotactile interaction on the distance of the stimuli. They used the visuotactile congruency task (see the next paragraph in the main text) to compare the condition where visual distractors were placed near the hands but seen as mirror reflections (i.e., seemingly distant visual images) with the condition where visual distractors were actually presented at far positions from the hands. They observed a larger congruency effect in the former condition than the latter, implying greater visuotactile interactions in the near than far space. Holmes and his colleagues also analyzed the extension of peripersonal space during tool-use, but they did not directly show the dependency of visuotactile interaction on the distance of the stimuli $[23,24]$. Another index for the peripersonal space in humans is the hand-position dependent modulation of visuotactile interaction, because single cell recordings in monkeys revealed that the visual receptive field of bimodal visuotactile neurons was anchored not to eye position but to arm position (e.g., [20]). Spence, Pavani and Driver ([53], Experiment 3) psychophysically demonstrated this phenomenon using the visuotactile congruency task.
} 
distance between visual and tactile stimuli. There may be at least two possible reasons for their failure to confirm the visuotactile peripersonal space. The first is that they used a relatively short distance in the experiment. In Sambo and Forster [46], visual stimuli in the "far" condition were presented at a distance of $70 \mathrm{~cm}$, while Forster et al. used a distance of $28.5 \mathrm{~cm}$ from the hands [10]. Single cell recordings in monkeys reported that responses of visuotactile bimodal neurons to visual stimuli are stronger when the distances between the hands and the visual stimuli are shorter. Some F4 neurons respond only to visual stimuli that are close to the body surface (less than $10 \mathrm{~cm}$ away), while others can be triggered by visual stimuli located further away but which is at a distance that is within the animal's reach $[13,14,45]$. Visuotactile crossmodal extinction occurred when visual stimuli were presented near the patient's ipsilesional hands $(\sim 5 \mathrm{~cm})$, but did not occur when visual stimuli were presented away from the hands $(\sim 35 \mathrm{~cm})$. Thus, possibly, the distance of $28.5 \mathrm{~cm}$ used by Forster et al. might be too short to elucidate the distance dependency of visuotactile interactions [10]. The second reason is related to the task requirement. While Sambo and Forster adopted a go/no-go task with tactile pattern discrimination [46], Forster et al. used the simple detection task [10]. Several studies reported that task requirements affected the appearance of visuotactile interactions [16,22]. For example, Girard et al. demonstrated using RTEs that the spatial consistency between visual and tactile stimuli did not affect visuotactile interactions when participants were required to produce speeded responses to all stimuli irrespective of their spatial position (i.e., a simple detection task) [16]. In contrast, the effect of spatial consistency between visual and tactile stimuli was pronounced when they were required to respond to stimuli in the right hemispace and ignore the stimuli appearing in the left hemispace (i.e., a go/no-go task). Thus, the present study adopted a go/no-go task, a more demanding task that can measure RTEs, rather than a simple detection task (the details have been described in Experiment 2).

The present study investigated how the difference in the depth of the visual and tactile stimuli affected visuotactile interactions using the crossmodal congruency effects in Experiment 1 and the RTEs in Experiment 2. Both experiments demonstrated the existence of the peripersonal space in healthy humans.

\section{Experiment 1}

The present study introduced a simpler version of speeded discrimination task (i.e., left/right discrimination instead of up/down elevation discrimination), since the manipulation of the distance between the visual and tactile stimuli was likely to make the up/down elevation discrimination task more difficult than usual. Participants discriminated the location of vibrotactile target stimuli presented on their left or right hand while they tried to ignore visual distractors that were independently presented close to or away from the tactile stimuli, either on the same side as the target stimuli or on the opposite side. Several studies have used this type of crossmodal congruency task (i.e., left/right discrimination) to investigate differences in audiotactile interactions between near and far spaces $[27,55]$.

\subsection{Methods}

\subsubsection{Participants}

Ten undergraduate students (aged 20-22 years, mean age $21.4 \pm 0.7$ years; all males) took part in this experiment. All the participants were right-handed and had normal or corrected-to-normal visual acuity. This study followed the tenets of the Declaration of Helsinki. Participants provided their informed consent to participate in this study before commencement of the experimental sessions.

\subsubsection{Apparatus and Stimuli}

The participants sat in a dimly lit experimental chamber resting their hands on a table in front of them. They put their left and right index fingers on vibrotactile stimulators (Eishin Denki K.K., M-PZT-02) placed $22 \mathrm{~cm}$ to the left and right of their midline, respectively, at a distance of approximately $50 \mathrm{~cm}$. Two sets of two white LEDs (light emitting diode, $3 \mathrm{~mm}$ in diameter) were each placed $22 \mathrm{~cm}$ to the left and right of their midline at a distance of $5 \mathrm{~cm}$ (near) and $70 \mathrm{~cm}$ (far) from the vibrotactile stimulator on each side, respectively (Fig. 1). A red fixation cross was placed at the center of the table, equidistant from the white LEDs. Participants were required to keep looking at the fixation point throughout the experimental session and not allowed to directly see their hands. In this situation, participants were able to clearly see all LEDs, while their hands were faintly visible. The vibration amplitude was set far above the detection threshold, at a frequency of $300 \mathrm{~Hz}$. The duration of the visual and tactile stimuli was $100 \mathrm{~ms}$. The synchronization of the onset of the visual and tactile stimuli was confirmed using a digital oscilloscope. The participants' left and right feet were placed on the left and right foot pedals, respectively, and they responded by pressing these pedals. The experiment was controlled by an IBM-compatible personal computer (Dospara, Prime PC). Digital signals for the LEDs and vibrotactile stimulators were converted to analog using audio interfaces (Roland, Octa-capture, U-1010). To mask any noise made by the operation of the vibrotactile stimulators, a white noise was presented continuously at $68 \mathrm{~dB}$ (A-weighted sound pressure level) via headphones, throughout the experiment. We confirmed that all the participants did not hear any operation noise over the masking noise. 


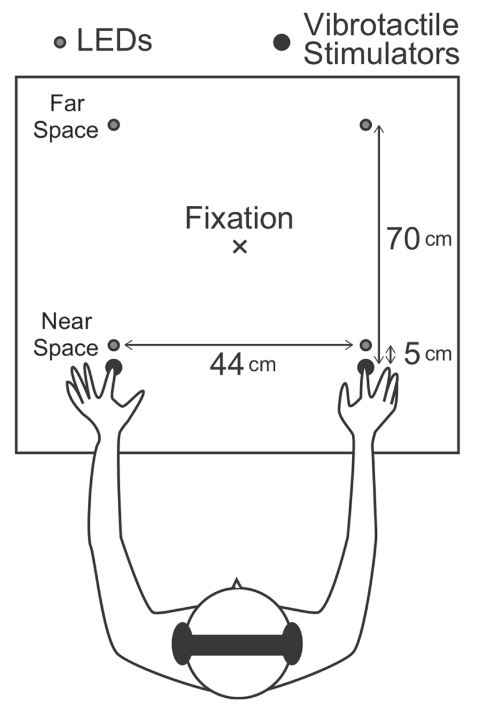

Fig. 1. Schematic illustration of the experimental setup (top view). Participants put their left and right index fingers on left and right vibrotactile stimulators, respectively. Visual stimuli were presented on the four possible locations in the near and far space in the two hemispaces. To mask any noise made by the operation of the vibrotactile stimulators, a white noise was presented continuously at $68 \mathrm{~dB}$ SPL(A-weighted) via headphones, throughout the experiment.

\subsubsection{Procedure}

Participants were instructed to produce speeded left/right discrimination responses regarding the side from which the tactile targets were presented, ignoring the visual stimuli. The participants depressed the foot pedal that corresponded to the side on which the tactile target was presented. Each participant completed one experimental session of 170 trials: 2 tactile conditions (left and right) $\times 5$ visual conditions (4 positions of visual distractors and no visual stimulus) $\times 15$ repetitions, plus 20 catch trials (4 positions of visual distractors without tactile targets $\times 5$ repetitions). Trials in which a target and distractor were presented on the same side were defined as the congruent condition, while those in which they were presented on opposite sides were defined as the incongruent condition. Further, trials in which no visual distractor was presented were called as the baseline condition. The order of these conditions was randomized. The inter-trial interval was randomly chosen from $1000 \mathrm{~ms}$ to $1500 \mathrm{~ms}$. Before the experimental session, each participant completed one practice session of 20 trials to enable the experimenter to confirm if they understood how to do the task.

\subsubsection{Data analysis}

To investigate any potential speed-accuracy trade-offs, we calculated "inverse efficiency (IE)" scores. IE scores are a standard way to combine RT and accuracy data into a single performance measure, computed as the median RT divided by the proportion of correct trials for a given condition $[27,58]$. A higher IE value indicates worse performance, just as for RT and error measures.

\subsection{Results and Discussion}

Trials in which the RTs were less than $100 \mathrm{~ms}$ and more than $1000 \mathrm{~ms}$ were discarded from the following analysis as errors. The error rate was $0.5 \%$. Trials with an incorrect response were also discarded from the analysis of the RT data. The rates of incorrect responses were $2.4 \%$ for the baseline (tactile only) condition, $0.0 \%$ and $6.3 \%$ for the near congruent and incongruent conditions, respectively, and $1.0 \%$ and $0.3 \%$ for the far congruent and incongruent conditions, respectively. Figure 2 shows the average IE scores for each condition. To investigate whether the visual distractors affected the tactile left/right discrimination performance, one-way analysis of variance (ANOVA) (near vs. far vs. baseline) was conducted for the IE scores for each of the congruent and incongruent conditions. A main effect of visual distractors was significant $\left(F_{2,18}=11.21, p<.001, \eta^{2}=.197\right.$ for the incongruent condition and $F_{2,18}=16.73$, $p<.001, \eta^{2}=.110$ for the congruent condition). The multiple comparisons (Tukey's HSD, $\alpha<.05$ ) revealed that the IEs were significantly larger for the near condition than for the other conditions in the incongruent trials (interference effect). In the congruent trials, the IEs were significantly smaller for the near condition than for the other conditions (facilitation effect). Thus, the visual distractors influenced the tactile left/right discrimination performance only in the near condition. This indicates the existence of the visuotactile peripersonal space.

\section{Experiment 2}

Experiment 2 used a go/no-go task and investigated whether the RTEs were affected by the space where visual 


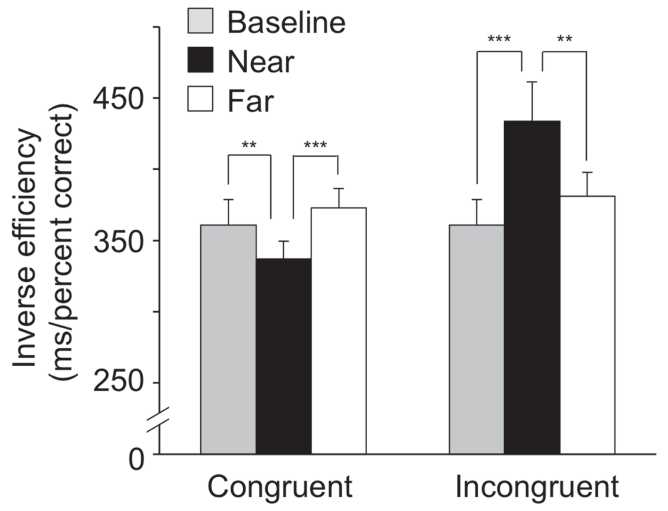

Fig. 2. Results of Experiment 1. Averages of inverse efficiency scores (i.e., median RT/proportion of correct responses) across participants $(N=10)$ are shown for each condition. Visual and tactile stimuli were presented in different hemispaces in the incongruent trials, while they were presented in the same hemispace in the congruent trials. The data for the baseline condition were identical between the congruent and incongruent conditions. The error bars represent the within-participants standard error of mean. Asterisks indicate significant differences between the conditions, $*: p<.05, * *: p<.01, * * *: p<.001$.

stimuli were presented. The violation of the race model only in the proximal space of the hands would indicate the existence of visuotactile peripersonal space in healthy humans.

\subsection{Methods}

The ten undergraduate students (aged 20-22 years, mean age: $21.4 \pm 0.7$ years; all males) took part in this experiment. The participants were required to produce speeded responses to all stimuli appearing in one hemispace (left or right), irrespective of their spatial position (near/far) and modality (visual/tactile/visuotactile). Before the experimental session, the experimenter informed the participants about the side on which they had to respond. In several trials, the tactile stimuli appeared on the other side, but the participants were required to refrain from responding to these trials (no-go trials). Responses were made by depressing a foot-pedal placed under the participants' dominant foot. Each participant completed two experimental sessions: in which the go trials were on the left side and in which the go trials were on the right side. The order of the sessions was counterbalanced among the participants. Each session consisted of 64 trials: 8 for each of the tactile only (T), visual only (V; near and far) and visuotactile (VT; near and far) conditions and 24 for the no-go trials. The order of these conditions was randomized. The inter-trial interval was randomly chosen from $1000 \mathrm{~ms}$ to $1500 \mathrm{~ms}$. Before the experimental sessions, each participant completed one practice session of 20 trials to enable the experimenter to confirm if they understood how to do the task. Participants were required to keep looking at the fixation point throughout the experimental session and not allowed to directly see their hands. In this situation, participants were able to clearly see all LEDs, while their hands were faintly visible.

\subsection{Results and Discussion}

Trials in which the RTs were less than $100 \mathrm{~ms}$ and more than $1000 \mathrm{~ms}$ were discarded from the following analysis as errors. The error rates were $2.5 \%$ for the tactile only condition, $6.3 \%$ and $5.0 \%$ for the near visual and visuotactile conditions, respectively, and $2.5 \%$ and $5.0 \%$ for the far visual and visuotactile conditions, respectively. The false alarm rate in catch trial was 7.1\%. A one-way ANOVA for the error rates revealed no main effect $\left(F_{4,36}=1.28, p=.298\right.$, $\left.\eta^{2}=.074\right)$. Figure 3 shows the average RTs for each condition. Note that the average RTs for the tactile only condition (gray bars) were identical between the near and far conditions. A one-way ANOVA was conducted on the RT data ( $\mathrm{T}$, $\mathrm{V}$, and VT) for each of the near and far trials. A main effect of condition was significant, irrespective of the near and far trials $\left(F_{2,18}=37.81, p<.001, \eta^{2}=.313\right.$ for the near trials and $F_{2,18}=13.55, p<.001, \eta^{2}=.247$ for the far trials). The multiple comparisons (Tukey's HSD, $\alpha<.05$ ) revealed that, in the near trials, the RTs were significantly shorter for the VT condition than for the others. In the far trials, the RTs were significantly shorter for the T condition than the $\mathrm{V}$ condition, and much shorter for the VT condition than the other conditions. These results indicated that visuotactile redundant targets facilitated the detection performance, irrespective of the distance of the visual target. but if essential they must be defined at their first mention in the abstract itself.

Both the race and coactivation models can explain the data at this analysis stage. Thus, in the next step, we investigated whether the RT for each of the near and far visuotactile conditions exceeded the statistical facilitation predicted by probability summation using Miller's race model of inequality [37], using a MATLAB code described in [59]. The procedure was as follows: Firstly, two cumulative probability density functions (CDFs) of the RTs were estimated for the VT and for the sum of the $\mathrm{V}$ and $\mathrm{T}(\mathrm{V}+\mathrm{T})$ conditions, respectively. Subsequently, RT values for each condition were calculated at 17 points corresponding to the 10th through 90th percentiles with bin width of $5 \%$. This procedure was applied for each participant's data and the average CDFs across the participants were calculated. Finally, for each percentile, the VT and V $+\mathrm{T}$ were compared using a two-tailed t-test. These calculation procedures were 


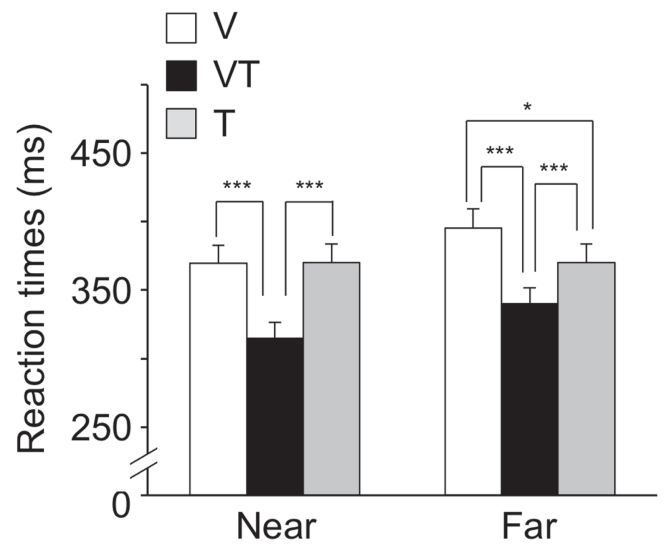

Fig. 3. Average reaction times across participants $(N=10)$ for the visual stimulus only $(\mathrm{V})$, tactile stimulus only (T), and visuotactile (VT) trials in the near and far conditions in Experiment 2. Asterisks indicate significant differences between the conditions, $*: p<.05, * *: p<.01, * * *: p<.001$. Redundant target effects were observed both for the near and far conditions. The data for the tactile only condition were identical between the near and far conditions. The error bars represent the within-participants standard error of mean.

(a) Near

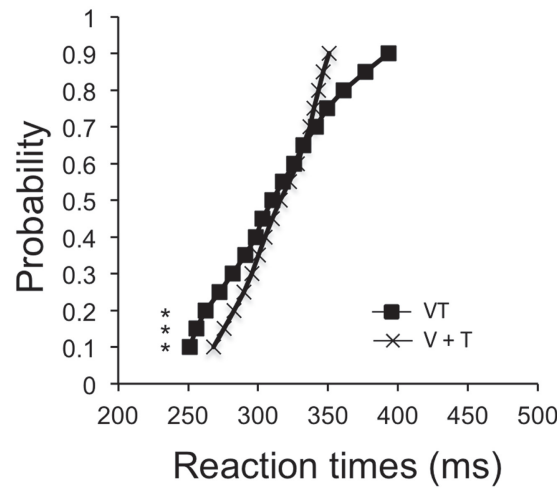

(b) Far

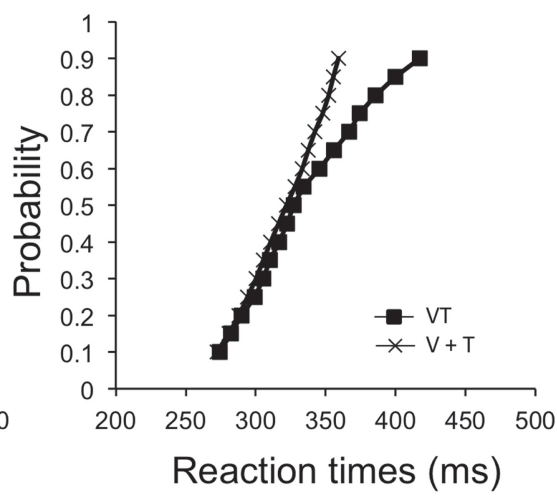

Fig. 4. Cumulative probability distribution functions for responses to visuotactile stimuli (VT) and the sum of responses to unisensory stimuli $(\mathrm{V}+\mathrm{T})$ in the near (a) and far (b) conditions in Experiment 2. Asterisks indicate significant differences between the conditions.

applied for each of the near and far conditions. Figure 4 shows the CDFs for the VT and V $+\mathrm{T}$ conditions (Figure 4(a) for the near condition and Figure 4(b) for the far condition). Separate comparisons were made for the first five percentiles using a one-tailed t-test with Bonferroni correction (significant level: $p<.05$ ). Significant violation of the race model prediction was observed for 10th, 15th, and 20th percentiles ( $t_{9}=3.35$ to $t_{9}=4.24, p=.008$ to $\left.p=.002\right)$ in the near condition; but the same was not observed in the far condition. This was consistent with the results of Experiment 1 , indicating that the visuotactile peripersonal space is distinctly represented in healthy adults as was exhibited in monkeys and patients with RBD.

\section{General Discussion}

The present study investigated whether the visuotactile peripersonal space is distinctly represented in healthy human adults, using psychophysical methods. Experiment 1 showed that the visuotactile crossmodal congruency effects, irrespective of the facilitation and interference effects, were salient only when visual stimuli were presented in proximity to the hands. Further, using the go/no-go paradigm, Experiment 2 demonstrated that the visuotactile RTEs that occurred near the hand violated the race model prediction, but those that occurred away from the hand did not. These results indicate that the visuotactile peripersonal space is distinctly represented in healthy adults.

Single cell recordings in monkeys first revealed the discrete processing of peripersonal space in brain areas such as the putamen, parietal, and premotor areas (e.g., $[3,9,13,18-20,45]$ ). The functional similarities in humans were mainly evidenced by studies on patients with RBD exhibiting extinction. However, there has been only one study that investigated whether the spatial modulation of visuotactile interactions in the near-far dimension in healthy human adults [46]. Since Sambo and Forster used the tactile pattern discrimination paradigm that was optimized for the eventrelated potential (ERP) measurements [46], the extent to which their findings can be generalized in the visuotactile 


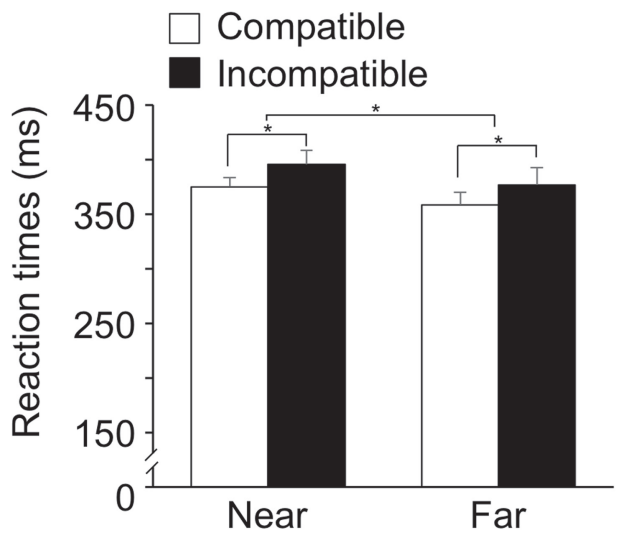

Fig. 5. Average reaction times across participants $(N=15)$ for the stimulus response compatibility effects (i.e., Simon effects) in the near and far conditions in the follow-up experiment. Stimuli were presented on the side opposite to the side on which the response button was assigned in the incompatible trials, while they were presented on the same side as response button in the compatible trials. Asterisks indicate significant differences between the conditions, $*: p<.05, * *: p<.01, * * *: p<.001$. The error bars represent the within-participants standard error of mean.

interaction literature is unclear. The present study used two psychophysical measurements that have normally been utilized in multisensory research. Thus, it provided new behavioral evidence that the space immediately around a body part is specially represented in brains of healthy humans as well as of monkeys and patients with RBD. Recent neuroimaging studies that involved manipulation of the distance of visual stimuli from body parts demonstrated that the visual space near body parts is distinctly represented in several brain areas of healthy adults, such as the intraparietal sulcus, the lateral occipital complex, and premotor cortex (e.g., [4, 12,35]; see also [7,34] for a review). Our behavioral findings would support the findings of these neuroimaging studies.

As for the visuotactile congruency effects observed in the present study, there are at least 3 potential causes [47, 54]: crossmodal exogenous attention, response conflict, and perceptual interaction. With reference to crossmodal exogenous attention, the presentation of the visual distractor captured the exogenous attention of the participants to the distractor side, which could have resulted in slower responses to tactile targets in the incongruent condition, and faster responses in the congruent trials, than in the baseline (tactile only) condition (e.g., $[25,47,50,51])$. The difference between the near and far visual conditions might be explained by greater exogenous attentional cueing effects in the visual space near the hands, rather than away from them. Previous studies suggested that crossmodal exogenous attention is evident when the visual distractor preceded the tactile target by approximately $100-300 \mathrm{~ms}$ (e.g., $[25,47,54])$. This range is different from our experimental setting where the onsets of the visual distractor and tactile target were synchronized. If exogenous attentional cueing triggered by visual stimuli influences task performance only when the stimuli are presented within that range, this perspective might be an unlikely explanation for the current results. However, recent studies showed that there are attentional biases near the hands (e.g., [40,41]). This suggests a possibility that some attentional mechanisms involve the current phenomenon. Further experiments are required to clarify this point.

The response conflict perspective argues that the crossmodal congruency effects reflect the consequences of competition between the response tendencies elicited by the visual distractor and tactile target (cf., [49]). Visual and tactile stimuli might prime the response on the different sides, resulting in more errors and greater RTs for the incongruent trials than for the baseline trials. In contrast, in the congruent trials, since both visual and tactile stimuli primed the response on the same side, the responses were more accurate and quicker than those in the baseline trials. This would suggest that visuotactile interactions occurred at a response selection level. The effect of distance might be explained by assuming more response priming for the visual stimuli near the hands rather than far from them. To test the hypothesis that the visual stimuli near the hands would have more response priming effects than the visual stimuli away from the hands, a follow-up experiment on stimulus-response compatibility was conducted (i.e., Simon paradigm, [48]). Each of the four white LEDs (2 for near locations and 2 for far locations) used in Experiments 1 and 2 was replaced with a pair of red and green LEDs, and the tactile stimuli were removed. In this experiment, one of the 8 LEDs was flashed for $100 \mathrm{~ms}$. The participants' task was to produce speeded responses to the color of the LED, irrespective of its location. When the red LED was flashed, participants were required to press the left foot-pedal (and the right footpedal when the green LED was flashed), as quickly as possible, irrespective of the location of the LED. If the visual stimuli near the hands would have had more response priming effects than the visual stimuli away from them, the stimulus-response compatibility effects should have been more salient when the closer rather than the farther LED was flashed. Contrary to this prediction, there were no differences in the magnitude of the stimulus-response compatibility effect between the near and far conditions (i.e., no interaction between space (near/far) and compatibility (compatible/ incompatible), $F_{1,14}=0.01, p=.914$, Figure 5). This result might imply that the response conflict account cannot fully explain the current results. 
In the perceptual interaction account, the visual distractor is considered to perceptually capture the location of the tactile stimuli, which could result in more errors and greater RTs for the incongruent than for the baseline trials [54]. Several studies have exhibited the visual capture of tactile perception (e.g., [2,5]) and facilitation of tactile perception when visual information was presented (e.g., [26,56]). Further, studies using a simple detection task as well as Experiment 2 of the present study exhibited visuotactile crossmodal facilitation $[10,16]$. Considering these findings, visual stimuli near the hands might more frequently ventriloquize tactile targets and more greatly enhance neural responses to tactile targets than those away from the hands.

For the RTEs, it should be noted that, while Forster et al. failed to demonstrate the distance dependency of visuotactile interaction (the violation of the race model was observed both for the near and far conditions) [10], Sambo and Forster [46] and the present study successfully demonstrated that the visuotactile crossmodal facilitation occurred only in the near condition. As mentioned in the Introduction, there are at least two differences in the experimental settings between the former and latter studies, namely, the distance of the far condition and the task. The present study (and Sambo and Forster [46]) presented the visual stimuli at a distance of $70 \mathrm{~cm}$ in the far condition, while Forster et al. did so at a distance of $28.5 \mathrm{~cm}$ [10]. Our results suggested a possibility that a distance of $28.5 \mathrm{~cm}$ away from the hands may not have been far enough to observe the distance dependency of visuotactile interactions (i.e., both conditions in near space).

Further, the task requirement was another important difference. Hecht et al. reported that the multisensory facilitation is larger for a choice RT task than a simple RT task [22]. Girard et al. also showed that the spatial consistency between visual and tactile stimuli affected visuotactile interactions in a go/no-go task, but not in a simple detection task [16]. Sambo and Forster [46] and the present study found the existence of the distance dependency of visuotactile interaction using go/no-go tasks and a crossmodal congruency task, while Forster et al. observed visuotactile interactions irrespective of the distance of visual stimuli from the hands using a simple detection task [10]. It would thus be likely that more demanding tasks than simple detection are needed to trigger the distance dependency of visuotactile interactions. Since it is reasonable to consider that demanding tasks require the integration of information from more brain regions than a simple detection task would, this might indicate that relatively higher order processing, than that which occurs in the early sensory/perceptual processing stage, is involved in the representation of the visuotactile peripersonal space in healthy human adults. These considerations are in agreement with Sambo and Forster's ERP data (i.e., enhancement of ERPs in the near condition around $100 \mathrm{~ms}$ post stimulus) [46], which are not as early as the crossmodal modulation of very early ERPs that occur irrespective of spatial consistency of bimodal stimuli previously reported by several studies $(\sim 40-50 \mathrm{~ms}$ post stimulus; [15] for auditory-visual; and [11,38] for audio-tactile interaction).

Note that there is still a possibility that the differences in visual processing, rather than visuotactile interactions, between the near and far conditions have influence on the the RTEs of Experiment 2, because RTs in visual only trials were significantly smaller for the near than far conditions $\left(t_{9}=3.34, p<.009\right)^{\dagger}$. This might reflect differences in visibility of visual stimuli or in deployment of visual attention between far and near conditions. Since the fixation point was placed in the middle of the table, visual stimuli were presented in the upper and lower visual field in the far and near conditions, respectively. Effective visual field is generally larger for the lower than upper visual fields so that far visual stimuli might not have enough visibility to facilitate visuotactile interactions, although we confirmed that visual stimuli were visible in the preliminary experiment. Regarding the attention deployment, the advantage for the lower visual field in visual performance was sometimes interpreted in terms of finely-tuned attention in the lower visual field [21]. Recent studies also showed that there are attentional biases near the hands (e.g., [40, 41]). Furthermore, stimuli were presented in the near space in the three-fourth trials of a session. This manipulation might have influence on the deployment of visual attention somehow. Further studies are required to exclude the effect of these confounders and to clarify the genuine distance-dependency of visuotactile interactions and its processing stages.

In summary, the present study investigated how visuotactile interactions altered depending on the distance of visual stimuli from the hands by using crossmodal congruency effects that appeared in the visuotactile congruency task, and RTEs that are normally observed in simple/choice detection and go/no-go tasks. Consistent with the data reported from studies on monkeys and patients with RBD, visuotactile crossmodal effects were more pronounced when the visual and tactile stimuli were presented in proximity to, rather than away from the hands for both measures. These findings suggest the discrete representation of visuotactile peripersonal space in the brains of healthy humans.

\section{REFERENCES}

[1] Bender, M. B., Disorders of Perception, Springfield, IL: Charles C. Thomas (1952).

[2] Botvinick, M., and Cohen, J., "Rubber hands 'feel' touch that eyes see," Nature, 391: 768 (1998).

[3] Bremmer, F., Schlack, A., Duhamel, J. R., Graf, W., and Fink, G. R., "Space coding in primate posterior parietal cortex,"

${ }^{\dagger}$ Whichever model (race model vs. coactivation model) was applicable in Experiment 2 was greatly affected by the performance in the visual-only conditions, because the CDFs for the $\mathrm{V}+\mathrm{T}$ condition were calculated from the visual-only conditions and the tactile-only condition, which was identical between the near and far conditions. If the performance in the visual only trials is consistent between the near and far conditions, the difference in visuotactile interactions between the near and far conditions should be directly reflected in the difference in the CDFs between the VT and $\mathrm{V}+\mathrm{T}$ conditions and would be easily interpreted. 
NeuroImage, 14: S46-S51 (2001).

[4] Brozzoli, C., Gentile, G., Petkova, V. I., and Ehrsson, H. H., "fMRI adaptation reveals a cortical mechanism for the coding of space near the hand," Journal of Neuroscience, 31: 9023-9031 (2011).

[5] Craig, J. C., "Visual motion interferes with tactile motion perception," Perception, 35: 351-367 (2006).

[6] di Pellegrino, G., Làdavas, E., and Farnè, A., "Seeing where your hands are," Nature, 388: 730 (1997).

[7] di Pellegrino, G., and Làdavas, E., "Peripersonal space in the brain,” Neuropsychologia, 66: 126-133 (2015).

[8] Diederich, A., and Colonius, H., "Bimodal and trimodal multisensory enhancement: Effects of stimulus onset and intensity on reaction time," Perception \& Psychophysics, 66: 1388-1404 (2004).

[9] Duhamel, J. R., Colby, C. L., and Goldberg, M. E., "Ventral intraparietal area of the macaque: Congruent visual and somatic response properties," Journal of Neurophysiology, 79: 126-136 (1998).

[10] Forster, B., Cavina-Pratesi, C., Aglioti, S. M., and Berlucchi, G., "Redundant target effect and intersensory facilitation from visual-tactile interactions in simple reaction time," Experimental Brain Research, 143: 480-487 (2002).

[11] Foxe, J. J., Morocz, I. A., Murray, M. M., Higgings, B. A., Javitt, D. C., and Schroeder, C. E., "Multisensory auditorysomatosensory interactions in early cortical processing revealed by high-density electrical mapping," Cognitive Brain Research, 10: 77-83 (2000).

[12] Gentile, G., Petkova, V. I., and Ehrsson, H. H., "Integration of visual and tactile signals from the hand in the human brain: an fMRI study," Journal of Neurophysiology, 105: 910-922 (2011).

[13] Gentilucci, M., Scandolara, C., Pigarev, I. N., and Rizzolatti, G., "Visual responses in the postarcuate cortex (area 6) of the monkey that are independent of eye position," Experimental Brain Research, 50: 464-468 (1983).

[14] Gentilucci, M., Fogassi, L., Luppino, G., Matelli, M., Camarda, R. M., and Rizzolatti, G., "Functional organization of inferior area 6 in the macaque monkey. I. Somatotopy and the control of proximal movements," Experimental Brain Research, 71: 475-490 (1988).

[15] Giard, M. H., and Peronnet, F., "Auditory-visual integration during multimodal object recognition in humans: A behavioural and electrophysiological study," Journal of Cognitive Neuroscience, 11: 473-490 (1999).

[16] Girard, S., Collignon, O., and Lepore, F., "Multisensory gain within and across hemispaces in simple and choice reaction time paradigms," Experimental Brain Research, 214: 1-8 (2011).

[17] Girard, S., Pelland, M., Lepore, F., and Collignon, O., "Impact of the spatial congruence of redundant targets on within-modal and cross-modal integration," Experimental Brain Research, 224: 275-285 (2013).

[18] Graziano, M. S., and Gross, C. G., "A bimodal map of space: Somatosensory receptive fields in the macaque putamen with corresponding visual receptive fields," Experimental Brain Research, 97: 96-109 (1993).

[19] Graziano, M. S., Hu, X. T., and Gross, C. G., "Visuospatial properties of ventral premotor cortex," Journal of Neurophysiology, 77: 2268-2292 (1997).

[20] Graziano, M. S., Yap, G. S., and Gross, C. G., "Coding of visual space by premotor neurons," Science, 266: 1054-1057 (1994).

[21] He, S., Cavanagh, P., and Intriligator, J., “Attentional resolution and the locus of visual awareness," Nature, 383: 334-337 (1996).

[22] Hecht, D., Reiner, M., and Karni, A., "Multisensory enhancement: Gains in choice and simple response times," Experimental Brain Research, 189: 133-143 (2008).

[23] Holmes, N. P., "Does tool use extend peripersonal space? A review and re-analysis," Experimental Brain Research, 218: 273282 (2012).

[24] Holmes, N. P., Calvert, G. A., and Spence, C., "Extending or projecting peripersonal space with tools? Multisensory interactions highlight only the distal and proximal ends of tools," Neuroscience Letters, 372: 62-67 (2004).

[25] Kennett, S., Spence, C., and Driver, J., "Visuo-tactile links in covert exogenous spatial attention remap across changes in unseen hand posture," Perception \& Psychophysics, 64: 1083-1094 (2002).

[26] Kennett, S., Taylor-Clarke, M., and Haggard, P., "Noninformative vision improves the spatial resolution of touch in humans," Current Biology, 11: 1188-1191 (2001).

[27] Kitagawa, N., Zampini, M., and Spence, C., “Audiotactile interactions in near and far space," Experimental Brain Research, 166: $528-537$ (2005).

[28] Làdavas, E., "Functional and dynamic properties of visual peripersonal space," Trends in Cognitive Sciences, 6: 17-22 (2002).

[29] Làdavas, E., di Pellegrino, G., Farnè, A., and Zeloni, G., "Neuropsychological evidence of an integrated visuotactile representation of peripersonal space in humans," Journal of Cognitive Neuroscience, 10: 581-589 (1998).

[30] Làdavas, E., and Farnè, A., Neuropsychological Evidence for Multimodal Representations of Space Near Specific Body Parts, In Driver, J., and Spence, C. (Eds.), Crossmodal Space and Crossmodal Attention (pp. 69-98), New York: Oxford University Press (2004).

[31] Làdavas, E., and Farnè, A., "Visuo-tactilerepresentation of near-the-body space," Journal of Physiology Paris, 98: 161-170 (2004).

[32] Làdavas, E., and Serino, A., “Action-dependent plasticity in peripersonal space representations," Cognitive Neuropsychology, 25: 1099-1113 (2008).

[33] Làdavas, E., Zeloni, G., and Farnè, A., "Visual peripersonal space centred on the face in humans," Brain, 121: 2317-2326 (1998).

[34] Macaluso, E., and Driver, J., "Multisensory spatial interactions: A window onto functional integration in the human brain," Trends in Neuroscience, 28: 264-271 (2005).

[35] Makin, T. R., Holmes, N. P., and Zohary, E., "Is that near my hand? Multisensory representation of peripersonal space in human intraparietal sulcus," Journal of Neuroscience, 27: 731-740 (2007).

[36] Maravita, A., Spence, C., Sergent, C., and Driver, J., "Seeing your own touched hands in a mirror modulates cross-modal 
interactions," Psychological Science, 13: 350-355 (2002).

[37] Miller, J., "Divided attention evidence for coactivation with redundant signals," Cognitive Psychology, 14: 247-279 (1982).

[38] Murray, M. M., Molholm, S., Michel, C. M., Heslenfeld, D. J., Ritter, W., Javitt, D. C., Schroeder, C. E., and Foxe, J. J., "Grabbing your ear: Rapid auditory-somatosensory multisensory interactions in low-level sensory cortices are not constrained by stimulus alignment," Cerebral Cortex, 15: 963-974 (2004).

[39] Previc, F. H., "The neuropsychology of 3-D space," Psychological Bulletin, 124: 123-164 (1998).

[40] Reed, C. L., Grubb, J. D., and Steele, C., "Hands up: Attentional prioritization of space near the hand," Journal of Experimental Psychology: Human Perception and Performance, 32: 166-177 (2006).

[41] Reed, C. L., Leland, D. S., Brekke, B., and Hartley, A. A., "Attention's grasp: Early and late hand proximity effects on visual evoked potentials," Frontiers in Psychology, 4: 420 (2006).

[42] Rizzolatti, G., Fadiga, L., Fogassi, L., and Gallese, V., “The space around us," Science, 277: 190-191 (1997).

[43] Rizzolatti, G., Luppino, G., and Matelli, M., “The organization of the cortical motor system: New concepts," Electroencephalography and Clinical Neurophysiology, 106: 283-296 (1998).

[44] Rizzolatti, G., Matelli, M., and Pavesi, G., "Deficits in attention and movement following the removal of postarcuate (area 6) and prearcuate (area 8) cortex in macaque monkeys," Brain, 106: 655-673 (1983).

[45] Rizzolatti, G., Scandolara, C., Matelli, M., and Gentilucci, M., "Afferent properties of periarcuate neurons in macaque monkeys. II. Visual responses," Behavioural Brain Research, 2: 147-163 (1981).

[46] Sambo, C. F., and Forster, B., "An ERP investigation on visuotactile interactions in peripersonal and extrapersonal space: Evidence for the spatial rule," Journal of Cognitive Neuroscience, 21: 1550-1559 (2009).

[47] Shore, D. I., Barnes, M. E., and Spence, C., "Temporal aspects of the visuotactile congruency effect," Neuroscience Letters, 392: 96-100 (2006).

[48] Simon, J. R., "Reactions towards the source of stimulation,” Journal of Experimental Psychology, 81: 174-176 (1969).

[49] Simon, J. R., The Effects of an Irrelevant Directional Cue on Human Information Processing, In Proctor, R. W., and Reeve, T. G. (Eds.) Stimulus-Response Compatibility (pp. 31-86), Amsterdam: Elsevier Science (1990).

[50] Spence, C., McDonald, J., and Driver, J., Exogenous Spatial Cuing Studies of Human Crossmodal Attention and Multisensory Integration, In Spence, C., and Driver, J. (Eds.) Crossmodal Space and Crossmodal Attention (pp. 277-320), Oxford: Oxford University Press (2004).

[51] Spence, C., Nicholls, M. E., Gillespie, N., and Driver, J., "Cross-modal links in exogenous covert spatial orienting between touch, audition, and vision," Perception \& Psychophysics, 60: 544-557 (1998).

[52] Spence, C., Pavani, F., and Driver, J., "Crossmodal links between vision and touch in covert endogenous spatial attention," Journal of Experimental Psychology: Human Perception \& Performance, 26: 1298-1319 (2000).

[53] Spence, C., Pavani, F., and Driver, J., "Spatial constraints on visual-tactile cross-modal distractor congruency effects," Cognitive Affective Behavioral Neuroscience, 4: 148-169 (2004).

[54] Spence, C., Pavani, F., Maravita, A., and Holmes, N., "Multisensory contributions to the 3-D representation of visuotactile peripersonal space in humans: Evidence from the crossmodal congruency task," Journal of Physiology Paris, 98: 171-189 (2004).

[55] Teramoto, W., Nozoe, Y., and Sekiyama, K., "Audiotactile interactions beyond the space and body parts around the head," Experimental Brain Research, 228: 427-436 (2013).

[56] Tipper, S. P., Lloyd, D., Shorland, B., Dancer, C., Howard, L. A., and McGlone, F., "Vision influences tactile perception without proprioceptive orienting," Neuroreport, 9: 1741-1744 (1998).

[57] Todd, J. W., Reaction to Multiple Stimuli, New York: Science Press (1912).

[58] Townsend, J. T., and Ashby, F. G., Stochastic Modelling of Elementary Psychological Processes, New York: Cambridge University Press (1983).

[59] Ulrich, R., Miller, J., and Schröter, H., "Testing the race model inequality: An algorithm and computer programs," Behavior Research Methods, 39: 291-302 (2007). 